\title{
Do We Ruin the Moment? Exploring the Design of Novel Capturing Technologies
}

\author{
Ine Mols ${ }^{1,2}$ \\ i.mols@tue.nl
}

\author{
Mendel Broekhuijsen ${ }^{1,2}$ \\ m.j.broekhuijsen@tue.nl
}

Elise van den Hoven ${ }^{2,1,3,4}$

elise.vandenhoven@uts.edu.au

\author{
Panos Markopoulos ${ }^{1,2}$ \\ p.markopoulos@tue.nl
}

Berry Eggen ${ }^{1,2}$

j.h.eggen@tue.nl

\author{
${ }^{1}$ Eindhoven University of \\ Technology \\ Dept. of Industrial Design \\ P.O. Box 513, $5600 \mathrm{MB}$
}

Eindhoven, the Netherlands

\author{
${ }^{2}$ University of Technology \\ Sydney \\ Faculty of Design, \\ Architecture and Building \\ Sydney, Australia
}

\author{
${ }^{3}$ ARC Centre of Excellence in \\ Cognition and its Disorders
${ }^{4}$ DJAD, University of Dundee Dundee, UK

\begin{abstract}
By capturing our experiences we often strive to better remember them in the future. However, the act of media capturing also influences these same experiences in the present, an area which is underexplored. This paper describes a study with the aim to inform the design of novel media capturing strategies. Adopting an approach of defamiliarization based on intervention and reflection, we strive to gain insights in the influences of future capturing technologies on the experience of a day out. We conducted an exploratory study in which 28 students went on a day out and used a variety of capturing strategies. Individual and group reflections on the experience during this day identified several important aspects that media capturing influences: engagement, perception \& attention and social activity. The paper concludes with implications for design and proposes three potential future directions for media capturing, that instead of disturbing the moment enhance the experience.
\end{abstract}

\section{Author Keywords}

Media technologies, capturing, defamiliarization, user experience, interaction design, photography

\section{ACM Classification Keywords}

H5.m. Information interfaces and presentation (e.g., $\mathrm{HCI})$ : Miscellaneous.

\section{INTRODUCTION}

The sun sets after a lovely day at the beach, but the magical moment is broken as people take selfies with their back towards the setting sun; the queen shakes hand with distinguished individuals during an annual award ceremony and many of them are busy taking pictures with their phones rather than attending to her; a concert visitor

Permission to make digital or hard copies of all or part of this work for personal or classroom use is granted without fee provided that copies are not made or distributed for profit or commercial advantage and that copies bear this notice and the full citation on the first page. Copyrights for components of this work owned by others than ACM must be honored. Abstracting with credit is permitted. To copy otherwise, or republish, to post on servers or to redistribute to lists, requires prior specific permission and/or a fee. Request permissions from Permissions@acm.org.

OzCHI '15, December 07 - 10 2015, Melbourne, VIC, Australia

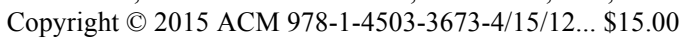
http://dx.doi.org/xx.xxxx/Xxxxxxx.xxxxxxx. misses the chorus of his favorite song as he is posting a movie of the first chords to his social network.

People will always be eager to capture their experience for later reminiscence. As the examples illustrate, mediatechnology can support us by capturing our experiences, but it can also get in the way of our experiences. As a natural tendency, people strive to both intensify and prolong positive emotional experiences, the latter being done through anticipation and reliving (Pohlmeyer, 2014). Media capturing is one way to enable reliving of our positive experiences. We often capture media to represent our experiences for affective reasons: to share the experience with others or for personal reminiscence Spasojevic et al., 2005). However, it seems that when we capture media with the purpose of reliving, the act of media capture itself can disrupt the very experience it intends to capture. At the same time, we could also consider capturing as a way of appreciating and participating in the moment (Banks, 2011), actively observing our surroundings or engaging in taking photos with other people. In other words, the influence that capturing of our experiences has on that moment is not straightforward. In this paper we explore that relation between capturing and experiencing, in order to inform the design of new and improved technologies for media capturing.

\section{Lived Experience}

HCI research that is looking at experience typically focuses on the user experience, where the experience is resulting from, or attributed to, the users' interactions with technology (Hassenzahl \& Tractinsky, 2006 ). In our exploration we approach experience from a more holistic perspective, including e.g. contextual, social and personal influences that are not attributed to the use of technology. To distinguish from user experience, we will refer here to the lived experience, in line with work done by e.g. McCarthy \& Wright (2005). Because of the unpredictable nature of the lived experience, and the emergent influence of both the involved technology and capturing strategy, we adopt an explorative approach focused on identifying the influence of a wide variety of capturing strategies on experiencing. Even though photography is the dominant form in everyday life, we use a broad definition of media capturing, including but not limited to taking photos, 
recording audio and video, writing text and collecting souvenirs. Media capturing practices are especially prevalent in tourism, where people seek to live positive experiences and very often capture such experiences for remembering or sharing. We therefore focus our exploration on the experience of a day out.

\section{Strategies for Capturing}

In our research we are interested in the influence of different capturing strategies on the experience of a day out. We focus on capturing strategies rather than technologies, because we refer to the appropriation of technology for media capturing within a specific context. There is an interplay between capturing technologies and the capturing strategies people use: certain technology enables or stimulates certain capturing strategies, e.g. the 'selfie' has increased in popularity since smartphones introduced front-facing cameras. As our primary interest lies with the variety in uses, we focus on strategies in our study.

In this paper we present a design-research study, exploring alternative capturing strategies to inform the design of future technologies. We used an interventionbased approach, to experience the influence of alternative capturing strategies and reflect on design implications. We will first discuss related work on common media capturing strategies and design interventions that have explored the influence of capturing on the lived experience. In the literature review we identify several characteristics of media capturing. In a field study with 28 students, we used these characteristics to develop alternative media capturing strategies. After describing the exploration, we reflect on which aspects of the experience are influenced by media capturing. Finally, we discuss implications for designing new media capturing technologies.

\section{RELATED WORK}

In this section we will discuss research related to the historical perspective of photo capturing and capturing technologies, followed by a brief overview of research into photo capturing strategies that have been identified to be of influence on the lived experience. We will mainly discuss photography, because of its dominance in social media and in touristic capturing practices, but mostly the examples apply at an abstract level to all media that can be used for capturing our experiences.

\section{History of Capturing}

The activity of capturing our experiences has been popular for decades, evidenced by all the letters, travel books, drawings, etc., that are preserved. After the invention of photography in 1830, the modern technology-mediated capturing slowly became accessible to the public, and by 1888 domestic photography was widely spread (Sarvas \& Frohlich, 2011). After decades of slow change, the introduction of digital photography brought many new opportunities: suddenly the limitations and costs of analogue photography were reduced radically, editing of photos became accessible, and sharing options expanded. With the introduction of smartphone technology with onboard cameras and internet access, the possibilities of capturing and instant sharing of experiences has expanded beyond photos, to include additional information such as location, tags of people that appear in the photo, and comments. This led to the omnipresence of capturing technologies in our current lives and to the question of their influence on our experiences.

\section{Capturing Experiences}

The influence of capturing our experiences can be understood from multiple perspectives. From a media perspective, taking a picture e.g. segments a portion of a scene in space and time, which represents the interest of the photographer. More interestingly, as has been explained by Gibson (1950), our perception is active: we perceive the world by interacting with it and within it, and influencing the interaction inevitably affects our perception of it. At a cognitive level, in line with the activity theory (Engeström et al., 1999), this can also be discussed in terms of the dual task that the photographer is engaging in: having to split attention between the surrounding events and scenes, and making a recording. Finally, at a social interaction level, the act of pointing a camera changes the experience for those who are recorded. Their actions take a more performative character. The social dynamics of the moment change as well as they are affected by the politics of ownership of the created record. In conclusion, striving to capture experiences, whether for social purposes, remembering purposes or any other purpose, will affect the experience in the moment in a variety of ways.

\section{Influence of Capturing on Experience}

An experiential perspective on media capturing could inform the design of media capturing technologies. However, most of the current capturing technologies have not been designed with that perspective in mind. At most, they focus on effective and usable capturing technologies. They consider the aesthetics of the interactivity without considering the effect of capturing on the experience itself. For example, Frens (2006) focuses narrowly on the affordances of mechanical elements of a photo camera, without embedding this interaction in the activity and social context of capturing a photo, ignoring embodiment (Dourish, 2004). When taking a user experience perspective, such a broader focus is required.

Other researchers have studied the effect of different capturing strategies on remembering or reliving events (Henkel, 2014; van den Hoven \& Eggen, 2009; Niforatos et al., 2014). They have investigated e.g. the influence of various modalities on the amount of detail in remembering an event (van den Hoven \& Eggen, 2009). But the influence of capturing these modalities has not been researched holistically. We therefore discuss several aspects of media capturing in both academic and commercial areas, which mention implications for the lived experience.

\section{The role of media type}

Although photos are the most prevalent media that people capture, technologies that offer capturing of different media are continuously being developed. Research efforts in e.g. capturing audio include Audiophotography, a combination of a normal photo augmented with either an 
environmental audio snippet, or audio commentary (Frohlich, 2004). In his book, Frohlich describes the experience of using Audiophotos to capture experiences. The participants who have tested the system over a longer period reported increased awareness to sounds in the environment (Forhlich, 2004). Another example of capturing alternative media has been developed by (Ljungblad et al., 2004), called context photography. In this media capturing application, sound and movement influence visual imagery. An exploration of this concept describes the influence of the application on the user experience as being experimental (Håkansson et al, 2006). Their exploration also shows how the modalities that are involved in media capturing influence how people perceive their environments, e.g. a passing car that would normally not have been noticed, suddenly becomes interesting to photograph (Håkansson et al, 2006). An interesting observation, making it worthwhile to further explore how different media types influence how we experience our environment.

\section{The role of frequency \& timing}

Digital photography has widely increased the quantity of media that we create, compared to analogue photos. Niforatos et al. (2014) proposed an experimental design to explore if limitations on digital pictures can result in pictures of higher memory value. Extreme examples that focus on almost unlimited capturing are the lifelogging devices, wearable cameras that automatically capture photos throughout the day at a given frequency (e.g. SenseCam, Hodges et al., 2006). In a long-term evaluation of using SenseCam, one of the participants indicated that in the beginning of the experiment, wearing the camera influenced his behavior, as he tried to do more interesting things, knowing that it would all be captured (Caprani et al., 2013). A commercial application that plays with the concept of timing is ROOM for thought (http://www.roomforthought.nl), which prompts once a day on a random moment to take a picture. For our focus, all these examples lead to the question how the frequency of media capturing influences the experience.

\section{The role of effort}

Related to automated capturing devices such as SenseCam, is the aspect of effort in capturing. The fact that SenseCam is making the photos automatically is important for the experience (Caprani et al., 2013). One of the participants in the long-term evaluation indicated that interacting with the camera would affect the experience, by interrupting it. Opposed to this type of effortless capturing, we see examples that describe the experience of effortful capturing, such as the work of Bakhshi et al. (2015) where participants are asked why they apply filters to their photos. Although it takes effort, this type of post-processing is making participants engage more with their photos, and the resulting media are more fun and special (Bakhshi et al., 2015). The need to obtain the same level of media but without putting in lots of effort is made possible to some extent by many commercial technologies that offer an easy way of applying filters or modifying photos (https://instagram.com). When it comes to aesthetics of media, people apparently have an idea of the effort a certain result should take: Ljungblad et al. (2004) found that if unintentional capturing leads to aesthetically pleasing media, participants tend to refer to it as cheating when they feel that they did not put enough effort in creating the end result. The question that is of interest is whether the limited effort and appreciation of the resulting media, also influences the experience in the moment itself.

To summarize: related work on media technologies mention different characteristics of these technologies that somehow influence the experience. Based on this review we decided that media type, frequency and effort are worthwhile to explore in this study.

\section{METHOD}

This study is a design-research exploration, focused on media technologies for remembering. To inspire novel directions, we need to go beyond observing current practices. People's attitudes towards capturing can be seen as being 'naturalized', as the cultural phenomena gradually became natural (Bell et al., 2005). People often do not critically think about how and why they capture. Similar to Bell et al. (2005), we therefore apply a process of defamiliarization in an attempt to estrange participants from regular practices, to encourage reflection on regular practices and as a way to identify novel directions for design. However, where Bell et al. approaches defamiliarization by changing perspectives through historical or ethnographic descriptions, we prefer to experience the unfamiliar. We therefore conducted an intervention-based study, concluded with reflection on current practices and opportunities for design. Based on a design-led and participatory mind-set (Sanders, 2008) we involved a group of 28 students as active contributors in this process.

\section{Study Design}

We conducted an exploratory intervention-based study: a field study in which participants explored alternative strategies during a day out. The study was designed to include multiple variables to explore a wide variety of capturing strategies, to gather as much information to inform future design. A set of 18 different strategies were developed and were deliberately distributed across participants to contrast with their current behavior. To inform this distribution a questionnaire was used to gather information on current behavior, as input for the process.

\section{Participants}

The study was run at Industrial Design faculty in Eindhoven with both undergraduate and graduate students participating. The study was part of the kick-off of a design project, as such the benefit of the excursion was twofold: the students explored the context of their new projects; we, as researchers had the opportunity to study and explore. This paper only discussed the latter.

The study was set-up with a participatory mind-set, involving the students throughout the process as contributors to the design insights. In total, 29 students participated in the activity, of which 28 consented to using their data for research. The participants, 12 male, 15 female, aged $18-25$, are all very familiar with common 
capturing technologies. Simultaneously, they studied interaction design at the time of this study, where they were involved in a project on designing novel media capturing technologies. As such, they approached the challenge both as users and as designers.

\section{Materials}

In this section we describe the materials and tools used for the study and provide more information on the alternative capturing strategies.

\section{Questionnaire}

To inform the development of alternative capturing strategies a questionnaire was used. The goal of the questionnaire was to get insight in the participants' regular media capturing strategies during days out. It included questions about their recent days out and how, in general, they created media during such days. Additionally, we asked about the technologies they used for this and what they thought to be the advantages and disadvantages of their strategies.

\section{Capturing strategies}

Based on current behavior we developed capturing strategies that were explicitly different. In order to achieve this, we looked in both the questionnaire and the literature for characteristics to change. As described, we selected media type, timing \& frequency, and effort, based on our review of related work. In addition to these aspects, we saw in the questionnaire that participants often used a specific focus on certain subjects in their current behavior. We therefore added subject as a variable, resulting in the following list of characteristic to explore in the alternative capturing strategies:

- The captured media type

- The amount of effort in capturing

- The timing \& frequency of capturing

- The subject of capturing.

We designed a wide range of strategies by varying these characteristics. For each participant a strategy was tailored to be different from his or her regular strategy in at least one of these characteristics. To enable this tailoring, the questionnaire was used to contrast the participants' regular capturing strategies.

We included eight different media types in our set of strategies, for 9 participants the media type was unchanged: photos. For all other participants the media type was changed (number of participants between brackets) audio (3), objects (2), data - GPS/subjective data (3), drawing (2), retrospective visual (2), video (4), words (3). These media types were combined with the other characteristics that were either changed or unchanged. Effort, timing and frequency of capturing were either drastically increased or decreased, compared to regular behavior. In some strategies a specific subject was prescribed, e.g. "other group members". This led to a total of 18 different strategies. The wide variety fitted the exploratory nature of the study. Because current behavior differs, this resulted in 28 unique cases, one for each participant. The cases were discussed and reflected on collectively and were not used for a comparative analysis. Space limits prevents us from presenting all 28 cases separately, however to provide insight in the process we summarize the strategies in Table 1, which gives an overview of the different categories and an example of current behavior to illustrate the change.

\section{Procedure}

The study procedure consisted of five parts (see Figure 1 for an overview): first, participants individually answered a questionnaire to bring regular behavior to their attention and inform the researchers on current behavior to inform alternative strategies. Secondly, participants received instructions on an alternative capturing strategy in one or two sentences (see Table 1) and including required materials (e.g. smartphone, pen and paper). Instructions were given one day in advance allowing participants to gather the required materials in time and start capturing from the start of their day. Thirdly, the participants experienced those strategies during a field trip to a nearby city. Most participants were familiar with the city but had not visited it 'as a tourist', only 2 participants were very familiar with or originated from the chosen city. Participants were encouraged to have, what they considered, a typical city excursion: they were given a self-guided city tour and were free to engage in other activities. Most participants explored the city in groups of 3 to 6 that they selected themselves. At the end of the day, the participants and the researchers gathered at the university to reflect on the experience. Researchers guided these reflections with prompts such as "What did you do differently?" "What did you perceive?". All participants gathered their insights and clustered these towards themes. The final step was done by participants individually, in the form of written reflections. These reflections included their experience with capturing, the resulting media, a design proposal for reliving these media and their insights. The data from observations, notes from the group reflections and the individual written reflections were used for thematic analysis.

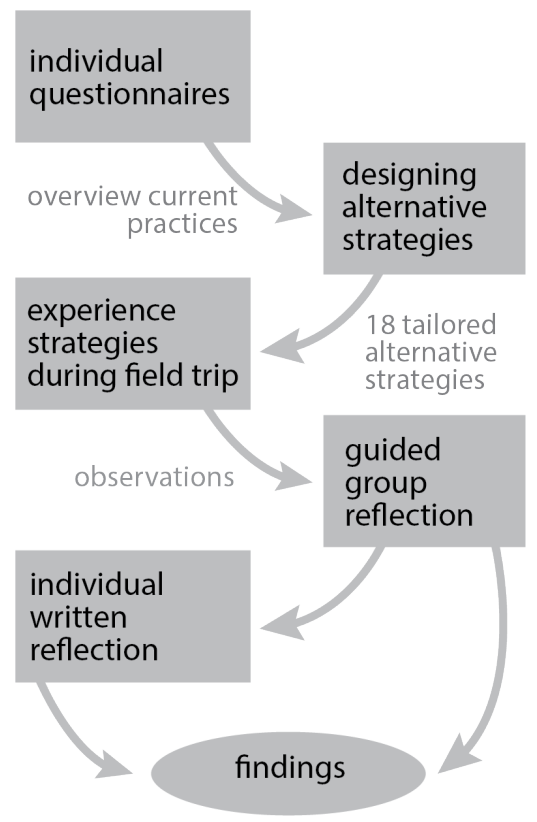

Figure 1: Visual overview of the study procedure. 


\begin{tabular}{|c|c|c|}
\hline $\begin{array}{l}\text { Changed strategy } \\
\text { characteristic }\end{array}$ & Example of regular capturing strategy & Alternative capturing strategy \\
\hline Frequency & $\begin{array}{l}{[\ldots] \text { most of the time I also take a few pictures of }} \\
\text { the people I'm with at that moment... I always try } \\
\text { to take a lot of pictures, but looking back I always } \\
\text { find that I did not take enough pictures of the } \\
\text { surroundings or buildings. [P7] }\end{array}$ & $\begin{array}{l}\text { Capture exactly } 1 \text { photo every hour [P7] } \\
\text { Capture as many photos as possible [P5] }\end{array}$ \\
\hline Media Type & $\begin{array}{l}\text { I often take photos because I think it's a very } \\
\text { quick, honest and good-looking way of recording } \\
\text { moments. [P19] }\end{array}$ & $\begin{array}{l}\text { Capture several spoken audio logs summarizing } \\
\text { events [P12, P19] } \\
\text { Collect many objects and souvenirs [P2, P11] }\end{array}$ \\
\hline Subject & $\begin{array}{l}\text { I tend to make a lot of funny photos and videos } \\
\text { with my friends. [...] so we can have a laugh a } \\
\text { few days later. [P15] } \\
\text { I do like it when people know that they are being } \\
\text { photographed or filmed, because in my opinion it } \\
\text { leads to the most fun and valuable material. [P14] }\end{array}$ & $\begin{array}{l}\text { Create a humoristic / serious video documentary } \\
\text { [P3, P15] } \\
\text { Capture non-posed, paparazzi-style photos only } \\
{[\mathrm{P} 4, \mathrm{P} 14]}\end{array}$ \\
\hline $\begin{array}{l}\text { Effort } \\
\text { Frequency }\end{array}$ & $\begin{array}{l}\text { I tend to think about the picture(s) I make. Often } \\
\text { return from a day out with no more than 4-5 } \\
\text { pictures. [P23] }\end{array}$ & $\begin{array}{l}\text { Create many effortless, point-and-shoot photos. } \\
{[\text { P20, P23] }} \\
\text { Create a few effortful photos [P20, P22] }\end{array}$ \\
\hline $\begin{array}{l}\text { Media Type } \\
\text { Effort }\end{array}$ & $\begin{array}{l}\text { At a city trip I make a lot of pictures of all the } \\
\text { beautiful buildings around me. Then I make } \\
\text { hundreds of pictures. [P16] } \\
\text { When I go on a day out I like to take a lot of } \\
\text { pictures [...] but often the pics turn out quite } \\
\text { boring [P24] }\end{array}$ & $\begin{array}{l}\text { Describe moments in text, when you would } \\
\text { normally take a picture. [P1, P18] } \\
\text { Automatically capture personal GPS data [P16] } \\
\text { Make small sketches throughout the day [P24, } \\
\text { P25] }\end{array}$ \\
\hline $\begin{array}{l}\text { Media Type } \\
\text { Subject }\end{array}$ & $\begin{array}{l}\text { I like to collect visual information throughout the } \\
\text { day out. [P6] } \\
\text { I like pictures of people in action, unaware of the } \\
\text { fact that I am taking this picture. [P26] }\end{array}$ & $\begin{array}{l}\text { Capture audio recordings of environmental } \\
\text { sounds [P6] } \\
\text { Make videos of your personal responses [P21, } \\
\text { P26] }\end{array}$ \\
\hline $\begin{array}{l}\text { Media Type } \\
\text { Timing \& Frequency } \\
\text { Effort }\end{array}$ & $\begin{array}{l}\text { I do not usually take a lot of pictures on a day } \\
\text { out; I try to focus on enjoying the moment itself. I } \\
\text { do however always enjoy looking back at } \\
\text { pictures; it makes it easier to relive the memory. } \\
\text { [P17] }\end{array}$ & $\begin{array}{l}\text { Automatically capture time-lapse video [P17] } \\
\text { Describe moments in text at random moments } \\
\text { triggered by an alarm [P27] } \\
\text { Capture nothing during the day, create a photo } \\
\text { collage of public images afterwards. [P9, P10] } \\
\text { Manually store GPS data and create panoramas, } \\
\text { triggered by an alarm [P28] }\end{array}$ \\
\hline $\begin{array}{l}\text { Media Type } \\
\text { Effort } \\
\text { Timing \& Frequency } \\
\text { Subject }\end{array}$ & $\begin{array}{l}\text { Often I do not make photos of the event itself } \\
\text { because it can ruin the experience. [P8] } \\
\text { I try not to see the day through a camera; I only } \\
\text { make photos or videos a few times during the } \\
\text { day. [P10] }\end{array}$ & $\begin{array}{l}\text { Create an experience graph, choose } 6 \text { parameters } \\
\text { to keep track of and mark them on the chart each } \\
15 \text { min. }[\mathrm{P} 8, \mathrm{P} 13]\end{array}$ \\
\hline
\end{tabular}

Table 1: Overview of alternative capturing strategies. In the left column the changed strategy characteristics are listed; the middle lists examples from current strategies, taken from the participants' questionnaires; in the right column the alternative capturing strategies are listed along with the participants that adopted them.

\section{FINDINGS}

We will present our findings based on the individual and group reflections by all participants. During the day out most participants explored the city in groups of 3-6. The researchers did not predefine these groups. Each participant within a group adopted a different strategy. We focus here on discussing the aspects of the experience that were influenced by the alternative strategies. To clarify why we choose to cluster our findings on experience aspects rather than the changed characteristics of the strategy we would like to emphasize the explorative nature of the study: a wide diversity of strategies was explored and often multiple characteristics were changed. Therefore, presenting findings for the different characteristics would result in speculation on their influence. Instead, we present our findings for each aspect of the experience that we observe to be influenced by the strategies, which was the focus of this study. In our discussion we will further speculate how the different characteristics relate to these experience aspects.

\section{Influencing Engagement}

The experience of a day out is strongly influenced by a person's engagement with the activities and surroundings. With some of the strategies, trying to capture an experience increased the engagement. This was for example seen with strategies that explicitly require participants to consider emotions, such as recording short 
videos of emotional expressions or keeping a detailed record of experiences for an experience graph. P21 adopted an alternative strategy of making short videos of her emotional response to her experiences, which was in contrast with her regular strategy of creating hardly any media. She reflects:

"During the experience you think more about the moment itself since you consider and think more about your emotions [to be able to capture them], this leads to a more intense experience." [P21]

Interestingly, strategies that enhanced an experience through reflection could at the same time disturb the experience because of the time and effort required for reflection. Especially strategies with high frequency or fixed timing reduced the engagement. This was caused by the many interruptions such a strategy caused, requiring complete engagement with capturing. Other strategies decreased the engagement with the experience by drawing too much focus on freely capturing throughout the day. One of the participants strived to create high quality photographs:

"It was weird to conclude that I was not enjoying the environment for myself but I was looking for things to photograph that [could] result in nice pictures. " [P22]

"I was more occupied with taking [many] pictures rather than the subject of the pictures. This closed me off from the experience sometimes. " [P23]

The second example shows that when striving for quantity rather than quality, participants could feel disengaged with the experience.

In short, people felt disengaged with the activity if capturing required too much time and effort. However, the media capturing strategies that required effort through reflection were appreciated for enhancing the experience, by increasing the awareness of emotions.

\section{Influencing Perception \& Attention}

Media capturing can direct our attention to certain aspects of our surroundings. Focusing on certain modalities or aspects was positive when it allowed people to observe things they might otherwise miss. This was most often mentioned for the audio modality, which led to a certain perceptual focus:

"I noticed that focusing on audio only gave me the ability to be very alert to all audio. I noticed things that I normally wouldn't have stored." [P06]
"I was paying more attention to objects that were insignificant, (e.g. sugar sticks, coasters) appreciating the value of these objects as I documented them." [P11]

The second quote illustrates that perceiving other things also led to appreciating them more. In some cases, focusing on a specific modality also caused people to miss things they would otherwise perceive, e.g. by focusing solely on visual elements environmental sounds, such as birds, were no longer perceived. Some strategies with a focus on a specific subject had a similar effect:

"The strategy [creating funny documentary] made you focus on funny details in the city, I almost didn't see any of the architecture that I normally look at." [P03]

On the other hand, modalities that required a personal interpretation, such as describing a scene in words or drawings, triggered people to perceive more details:

"Describing a scene in words makes you look at it with more attention and a greater eye for detail" [P18]

To summarize, perception of the environment was often directed by the media type and subject of the capturing strategy. This led to perceiving the surroundings in a different and sometimes surprising way.

\section{Influencing Social Activity}

Media capturing strategies also have an effect on group behavior, even when the strategies are all individually assigned. For example, the strategy of one participant to draw specific aspects of the day (see figure 2) served as conversation starter within their excursion group. Some strategies turned into true group activities, for example when group members acted in the scenes of videos or when the media was created together. This was most strongly seen in the strategy to collect small objects during the day:

"Collecting objects kept the whole group busy, it was tangible and easy to relate to. Together we selected each item, which means we were deliberately creating memories as a group. This resulted in a stronger cohesive group feeling and shared memories. " [P02]

An interesting social effect was seen with a participant who experienced the different strategies within a group as a division of responsibility, which made him more relaxed during the day:

"Focusing on audio only gave me a lot of stress relief since all the other media like photos and videos were not my responsibility. " [P06]
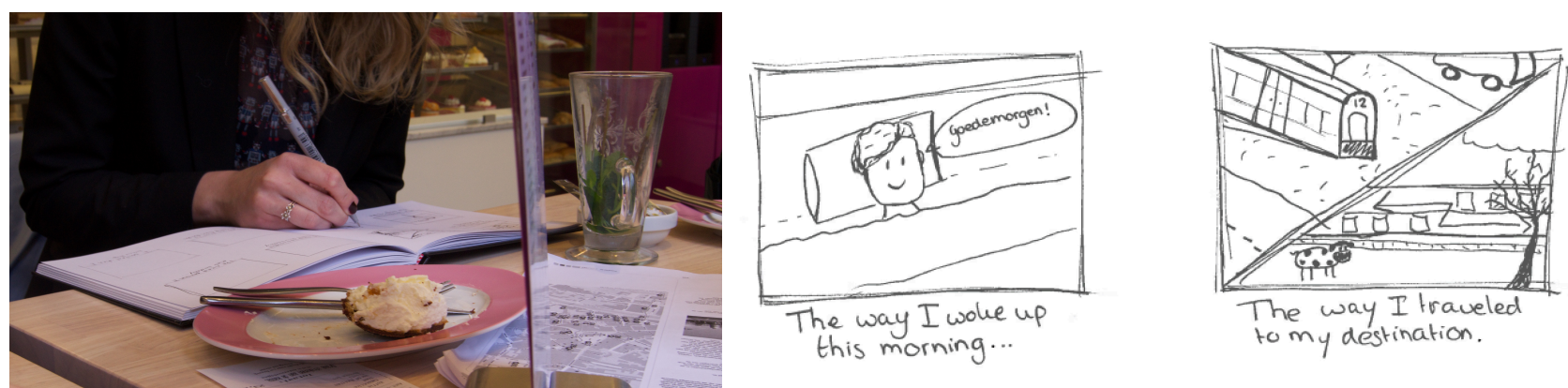

Figure 2: Example of a strategy changing all characteristics. Left: P25 drawing the day out (at lunch time). Right: example of the resulting media and predefined prompts 
However, the difference between individual strategies could also lead to friction: some capturing strategies isolated people from the group. In some cases, this was more through mental isolation, for example to describe a scene in words which is a very individual process. Other strategies required physical separation from the group, to better record environmental sound or video:

"As part of the serious documentary strategy I had to get nice and clear shots [of] the environment. To do so [...] I would either walk away from the group or ask them to step aside. I often became separated from the group. It felt like I was dragging behind sometimes and had to catch up. " [P15]

The negative effect of dragging behind or feeling like a burden was seen most in methods that took a lot of time, such as elaborately writing or shooting a video, see for example figure 3 .

To summarize, the influence of media capturing on the experience of a day out has been illustrated by the examples of the participants. In the following section we will discuss the implications on designing for novel media capturing strategies.

\section{DISCUSSION}

The exploration during a day out resulted in rich insights through the wide variety of strategies and the reflections from all participants.

\section{Limitations}

Participants' behavior during the staged day out differed in several ways from a 'normal' day out: the day was intended to explore capturing, while usually capturing is a side-activity. However, during the day people engaged in many 'normal' day out activities such as visiting the museum and having lunch. During all these activities capturing was a side-activity, but still received more attention than normally. Our insights do not provide conclusions regarding normal behavior, but through reflections can give direction for new opportunities. Moreover, in this study participants adopted a single strategy, which was very unnatural. More often people combine several strategies to fulfill their needs.

In our exploration, the day out was experienced in groups. Every member of the group adopted a strategy alternative to their regular capturing. In effect, participants influenced each other by e.g. triggering each other to capture certain things, or helping each other to capture unique media. This behavior is not completely different from usual group-activities, but with our exploration we observed more deliberate influences. The social nature of the day out also means that our findings do not address solo city trips.

\section{Strategy Characteristics}

The presented findings all relate to the adopted strategies and the aspects of frequency, timing, effort and media type. Due to the explorative nature of this study we are unable to isolate which characteristic causes which influence on the experience. Nevertheless, we would like to speculate on the influence of the separate aspects.

\section{Frequency}

Changing the frequency of capture was one of the characteristics with great influence, even if it was the only change. Unsurprisingly, predefined timing has a strong effect on engagement as alarms or triggers force you to interrupt the current activity. However, an advantage of fixed timing was the creation of more surprising media of otherwise uncaptured activities, such as traveling.

\section{Media type}

Participants that explored an alternative media type experienced the strongest novelty effects. Through the variety of media types we can see only one connection in this category: changing to an auditory modality seems to have the strongest and most positive effect on shifting perception. An observation that could be worthwhile to explore further.

\section{Subject}

Strategies that predefined a subject to capture had a similar effect in shifting perception. However, it was also one of the more difficult strategies for participants to adhere to as they often wanted to capture other subjects. In everyday use focusing on a certain subject is driven by personal interest and is applied far less rigid. Nevertheless, finding ways to suggest alternative subjects to capture through designed technologies could be a way to appreciate our experiences differently.

\section{Effort}

We observed that the amount of effort a strategy requires was the most important influence on all elements, perception, engagement and social interaction. It is important to recognize that 'effort' is not limited to the intended effort (e.g. create effortful photos) but is in itself an experience aspect that is influenced by many different characteristics of a strategy or technology, including media time and frequency.
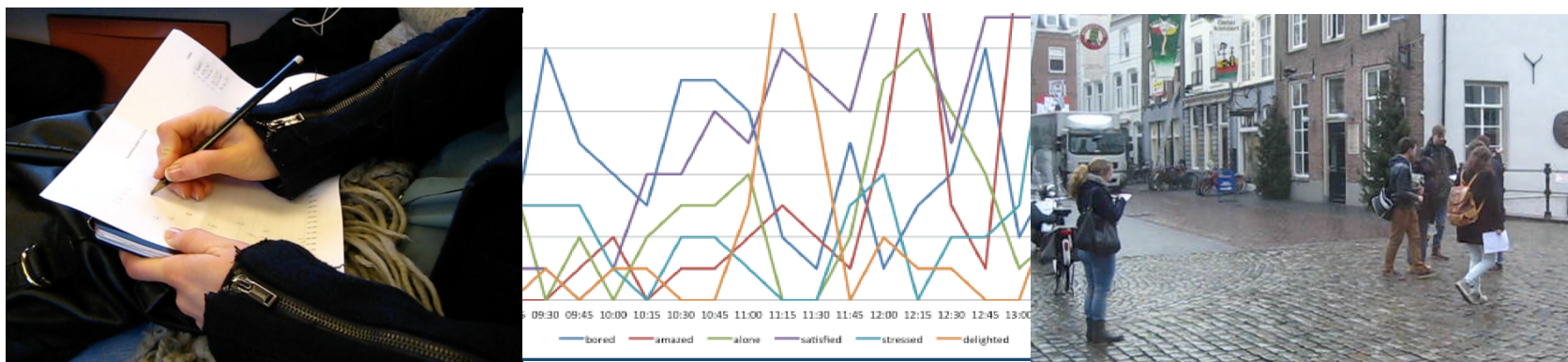

Figure 3: Examples of an alternative strategy. On the left: P13 creating an experience graph, making notes every 15 min on a graph for several experience aspects. In the middle: Resulting experience graph that was digitalized afterwards. On the right: social effect of the capturing strategy: being isolated from the group. 


\section{From Strategies to Technologies}

In this study, we prescribed specific strategies. It is important to notice that as designers we do not regularly design strategies but instead design technologies. These technologies can enable or steer towards certain aspects of the strategy but do not completely define it. Some aspects of a strategy are therefore difficult to implement in design because they relate more to specific use of the technology, e.g. by focusing on a certain subject to capture. In the following section we present the implications and opportunities derived from our strategies for the design of technologies.

\section{CONSIDERATIONS FOR DESIGN}

Before we provide suggestions for specific future directions, two general observations are important to consider when designing media capturing technologies. First of all, most of the characteristics were reported to have both positive and negative impact on the experience. Designers should therefore be aware of this trade-off. For example, the strategy of "making an experience graph" intensifies the experience (marked by the participant as being positive) but makes the person at the same time less engaged in a group. As a second remark we would like to emphasize the common practice of combining multiple strategies. People take, for instance, a few high quality pictures to decorate their home, take some snap shots to share on social media and save tickets for their collection. Each strategy serves a different need. When designing new capturing technologies, we should not try to replace all these strategies, but rather look for valuable additions to the spectrum of capturing possibilities. To do this we should focus on a specific contribution the technology can make in fulfilling people's needs.

We see three specific opportunities for novel media capturing design that can add value: design for perceptual focus, design for effortful creation, and social capturing.

\section{Directing Perceptual Focus Through Design}

The diversity in media types that we explored showed some interesting effects on the perceptual focus of the participants. The biggest influence was seen with recording audio, which changed their focus from visual perception to auditory perception. We see an opportunity to design media capturing technologies with explicit perceptual focus. Beyond modality, such technologies could stimulate us to perceive more details, observe patterns or discover hidden parts of our environment. Because it changes perception, designing with dedicated focus is especially suitable for specific scenarios. For example, making otherwise uninteresting aspects of a day more interesting (e.g. traveling, waiting). Or to rediscover a familiar location, e.g. enable people to be a tourist in their own city.

\section{Appreciation Through Effortful Creation}

In our findings we can distinguish two types of capturing effort. On the one hand some strategies involved the creation of high-threshold media such as writing, drawing or video blogging, which caused the participants to spend a lot of time and effort. On the other hand, some strategies implemented rules for the frequency of capturing, but used low-threshold media types. Especially the high-threshold media could have a positive effect on engagement by stimulating reflection. Current capturing technologies focus on making capturing easier and less effortful, but are often used frequently. It would be interesting to explore effortful creation to increase the appreciation of the experience. The effort should not be put in frequent, low-threshold capturing, which had more negative effects. Instead it could especially be done through creating technologies that allow freedom but require high effort, offering personal expression and interpretation. Such effortful practices can bring more value to people, and recent trends to e.g. choose vinyl records over mp3 illustrates that it is a promising direction for design.

\section{Design for Social Capturing}

With current technologies, many capturing strategies focus on single person use. Even if we are taking pictures of others, this is approached as an individual activity. An interesting direction would be to create more social capturing technologies that take into account that many of our leisure activities are enjoyed in the company of others. Creating media in collaboration rather than share individual media afterwards, can enhance these social experiences. Designs should focus here on a very specific social context: the needs for social capturing for couples are different from social capturing within groups of friends. However, when designing social capturing technologies, we should make sure people do not feel pressured or obligated to take the responsibility for the group if they do not want to. Instead design for social capturing could look at how the shared responsibility and shared activity can enhance the shared experience of going out together.

\section{CONCLUSION}

In this paper we presented an exploration in the design of new media capturing technologies and the influence it has on the experience of a day out. We reflected on the influence of alternative capturing strategies on different aspects of the lived experience, including the engagement, perception and social activity. We argue that designers can influence these experiences in the design of novel capturing technologies by carefully considering the impact of aspects such as frequency, media type, effort and subject. In addition, we see potential in three specific directions for novel media creation technologies: design to direct perceptual focus, design for effortful creation, and design for social capturing.

\section{ACKNOWLEDGMENTS}

This research was supported by STW VIDI grant number 016.128.303 of The Netherlands Organization for Scientific Research (NWO), awarded to Elise van den Hoven. The study was approved by the UTS Research Ethics Committee under UTS HREC REF NO. 2015000019. We would like to thank all the students for their willingness to participate and their honest reflections. We also thank the reviewers for their insightful comments and suggestions to help improve our paper. 


\section{REFERENCES}

Bakhshi, S., Shamma, D. A., Kennedy, L., \& Gilbert, E. Why We Filter Our Photos and How It Impacts Engagement. In Ninth International AAAI Conference on Web and Social Media. (2015).

Banks, R. The future of looking back. Microsoft Press, 2011.

Bell, G., Blythe, M., \& Sengers, P. Making by making strange: Defamiliarization and the design of domestic technologies. ACM Transactions on Computer-Human Interaction (TOCHI), 12,2 (2005), 149-173.

Caprani, N., O'Connor, N. E., \& Gurrin, C. Experiencing SenseCam: a case study interview exploring seven years living with a wearable camera. In Proceedings of the 4th International SenseCam \& Pervasive Imaging Conference ACM. (2013), 52-59.

Dourish, P. Where the action is: the foundations of embodied interaction. MIT press. (2004).

Engeström, Y., Miettinen, R., \& Punamäki, R. L. Perspectives on activity theory. Cambridge University Press. (1999).

Frens, J. A rich user interface for a digital camera. Personal and Ubiquitous Computing, 10(2-3) (2006) $177-180$

Frohlich. D. Audiophotography: Bringing photos to life with sounds, volume 3. Springer Science \& Business Media, (2004).

Gibson, J. The perception of the visual world. Houghton Mifflin, (1950).

Håkansson, M., Gaye, L., Ljungblad, S., \& Holmquist, L. E. More than meets the eye: an exploratory study of context photography. In Proceedings of the 4th Nordic conference on Human-computer interaction: changing roles ACM. (2006), 262-271

Hassenzahl, M., Tractinsky, N. User experience a research agenda. Behaviour \& Information Technology, 25(2), (2006), 91-97

Henkel, L.A. Point-and-shoot memories the influence of taking photos on memory for a museum tour. Psychological science, 25(2), (2014), 396-402
Hodges, S., Williams, L., Berry, E., Izadi, S., Srinivasan, J., Butler, A., ... \& Wood, K. DenseCam: A retrospective memory aid. In UbiComp 2006: Ubiquitous Computing. Springer Berlin Heidelberg. (2006), 177-193

Hoven, E. van den, Eggen. J.H. The effect of cue media on recollections. Human Technology: an International Journal on Humans in ICT Environments, 5(1), (2009)

Ljungblad, S., Hakansson, M., Gaye, L., \& Holmquist, L. E. Context photography: modifying the digital camera into a new creative tool. In CHI'04 extended abstracts (2004), 1191-1194

McCarthy, J. and Wright, P. Putting "felt-life" at the centre of human-computer interaction (HCI). Cognition, Technology \& Work 7, 4 (2005), 262-271.

Niforatos, E., Langheinrich, M., \& Bexheti, A. My good old kodak: understanding the impact of having only 24 pictures to take. In Proceedings of the 2014 ACM International Joint Conference on Pervasive and Ubiquitous Computing (2014), 1355-1360

Oleksik, G., Brown, L.M. Sonic gems: exploring the potential of audio recording as a form of sentimental memory capture. In Proceedings of the 22nd British HCI Group Annual Conference on People and Computers (2008), 63-172

Pohlmeyer, A.E. Enjoying joy: A process-based approach to design for prolonged pleasure. In Proceedings of the NordiCHI'14, (2014)

Sanders, L. On modeling an evolving map of design practice and design research. interactions, 15(6), (2008), 13-17

Sarvas, R., Frohlich, D.M. From snapshots to social media: The changing picture of domestic photography. Springer Science \& Business Media, (2011)

Kindberg, T., Spasojevic, M., Fleck, R., \& Sellen, A. The ubiquitous camera: An in-depth study of camera phone use. Pervasive Computing, IEEE, 4(2), (2005), 42-50 